\title{
Detection of Aedes aegypti larvae using single shot multibox detector with transfer learning
}

\author{
Mohamad Aqil Mohd Fuad ${ }^{1}$, Mohd Ruddin Ab Ghani ${ }^{2}$, Rozaimi Ghazali ${ }^{3}$, Tarmizi Ahmad Izzuddin ${ }^{4}$, \\ Mohamad Fani Sulaima ${ }^{5}$, Zanariah Jano ${ }^{6}$, Tole Sutikno ${ }^{7}$ \\ 1,2,3,4,5 Faculty of Electrical Engineering, Universiti Teknikal Malaysia Melaka (UTeM), \\ 76100, Durian Tunggal, Melaka, Malaysia \\ ${ }^{6}$ Centre for Languages and Human Development, Universiti Teknikal Malaysia Melaka (UTeM), \\ 76100, Durian Tunggal, Melaka, Malaysia \\ ${ }^{7}$ Department of Electrical Engineering, Faculty of Industrial Technology, Universitas Ahmad Dahlan (UAD), 3rd \\ Campus, Jln. Prof. Dr. Soepomo, Janturan, Yogyakarta 55164, Indonesia
}

\begin{abstract}
Article Info
Article history:

Received Jul 11, 2018

Revised Jan 7, 2019

Accepted Feb, 2019

Keywords:

Aedes Aegypti

Larvae detection

Multibox detector

ABSTRACT

The flavivirus epidemiology has reached an alarming rate which haunts the world population including Malaysia. In fact, World Health Organization has proposed and practised many methods of vector control through environmental management, chemical and biological orientations but still cannot fully overcome the problem. This paper proposed a detection of Aedes Aegypti larvae in water storage tank using Single Shot Multibox Detector with transfer learning. The objective of the study was to acquire the training and the performance metrics of the detection. The detection was done using SSD with Inception_V2 through transfer learning. The experimental results revealed that the probability detection scored more than $80 \%$ accuracies and there was no false alarm. These results demonstrate the effectiveness of the model approach.
\end{abstract}

Single shot

Transfer learning

\section{Corresponding Author:}

Rozaimi Ghazali,

Faculty of Electrical Engineering, Universiti Teknikal Malaysia Melaka (UTeM),

76100, Durian Tunggal, Melaka, Malaysia.

Email: dpdrudin@utem.edu.my
Copyright () 2019 Institute of Advanced Engineering and Science. All rights reserved.

\section{INTRODUCTION}

Advances in machine learning era has started when a classifier model has successfully trained using convolutional neural network architecture [1]. The reasons of the success are attributed to the computational power and the second one is due to a lot of database library for training purposes. Object detection is one of machine learning techniques. Basically, object detection is slightly different from classifier where it does not only find object inside the image, but also a pixel to localize the position of the object inside the image.

Dengue, chikungunya and yellow fever are acute febrile viral disease. The viruses are members of flavivirus family and have been transmitted by Aedes mosquitoes. In fact, World Health Organization has proposed and practised many methods of vector control through environmental management, chemical and biological orientations [2] yet they cannot fully overcome the problem. From the listed vector control, the most crucial part to be heeded are non-accessible places like water storage and artificial container [3]. This paper hence, proposed a detection of Aedes Aegypti larvae in water storage tank using Single Shot Multibox Detector with transfer learning. Therefore, the objective of the study was to acquire the training and performance metrics of the detection.

Lately, convolutional neural network has been a huge success in object detection. The first advance of machine learning in object detection is overfeat where the technique has proposed the sliding window algorithm using convolutional neural network to detect object [4]. However, the technique is very 
computational expensive. Just after the overfeat, region based convolutional neural network or R-CNN is published which triggers almost 50\% improvement [5]. The algorithm has proposed several approaches where it has created the bounding box using the region proposal method, features extraction using convolutional neural network, and classify the bounding box using support vector machine. This technique has rapidly evolved where Fast R-CNN [6] and Faster R-CNN [7] have yet to publish to improve the existing performance.

Shortly after the discovery of R-CNN, You Only Look Once or YOLO algorithm which use simple pipeline on yet convolutional neural network has resulted in high speed detection compared with Fast RCNN [8, 9]. Subsequently, Region-based fully convolutional network or R-FCN is developed, and this approach is likely to adopt Faster R-CNN architecture but with only convolutional networks [10]. Finally, Single shot multibox detector or SSD is developed to take on YOLO using the multiple size of convolutional feature maps which offer better results and speed in object detection [11].

Since most discoveries of viral disease are caused by the Aedes Aegypti, a lot of effort has been generated to combat this problem. Many methods are discovered including the use of neural network. Neural network has been used to identify the different of wingbeat frequencies of insects in flight [12]. Not just that, neural network has also been used to predict the day of defervesce of fever in dengue fever and dengue haemorrgic fever [13]. Besides, that backpropagation learning algorithm has also been used to predict and recognize dengue confirmed-case based on four important features-: mean relative humidity, total rainfall, mean temperature and total number of dengue confirmed-cases $[14,15]$.

\section{RESEARCH METHOD}

This section focuses on the development process of Aedes Aegypti larvae detection inside the water storage tank. The detection was done using SSD with Inception_V2 through transfer learning. SSD technique is based on feed-forward convolutional network and it is the first deep network pipe line based on object detector that does not need resample features or pixels for bounding box [11]. Figure 1 shows the architecture of SSD with Inception_V2 classifier.

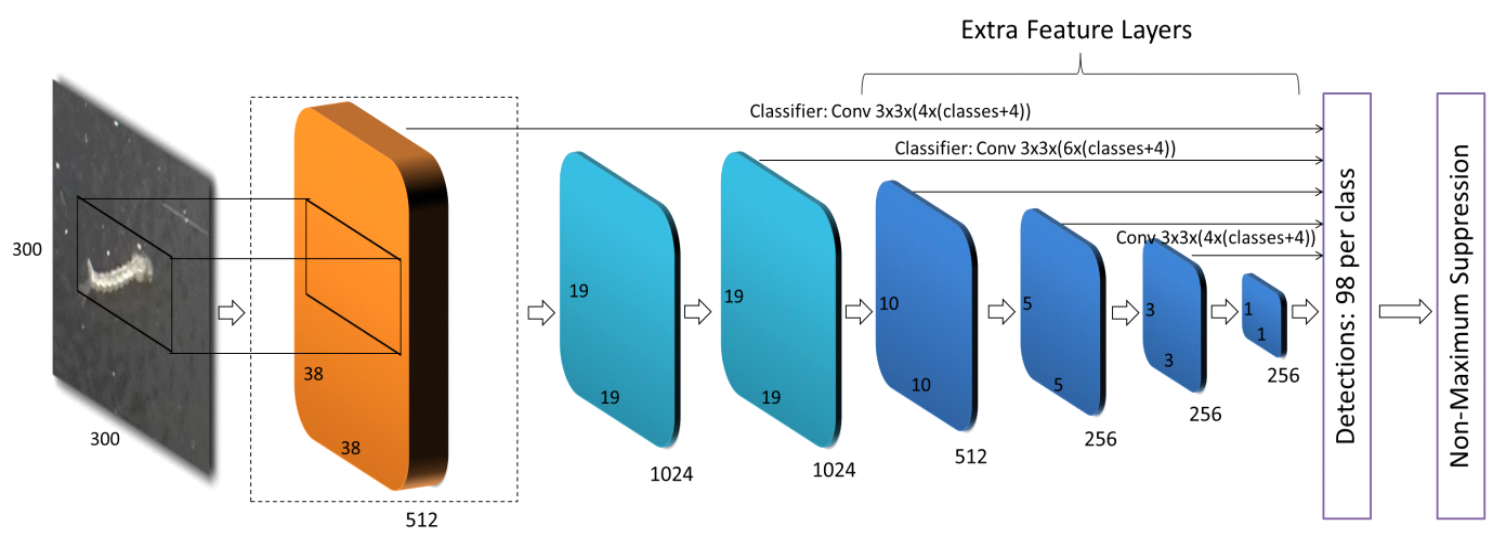

Figure 1. Architecture of SSD with Inception_V2 classifier

The first network layers were standard architecture used for image classification which also known as the base network. This experiment implemented the transfer learning where the inception_V2 was used for base network. Subsequently, the frame size and layers decreased progressively. In addition, each convolutional feature layer was truncated to detection layer, allowing multi-scale feature maps detection.

Transfer learning has improved accuracies and shown the tremendous classification in many and various object detection applications [16-19]. The dataset was first constructed to perform the transfer learning. The dataset was constructed by capturing the Aedes Aegypti larvae image during experiments in lab. The samples then were split into two groups to acquire the training and test patch images. About 176 images were created, then, the location of Aedes Aegypti larvae was labelled and identified. The trained model, then, was tested to measure the performance. Figures 2 and 3 shows the flowchart of the experiment and illustrate the default box procedure on detecting Aedes Aegypti larvae. 


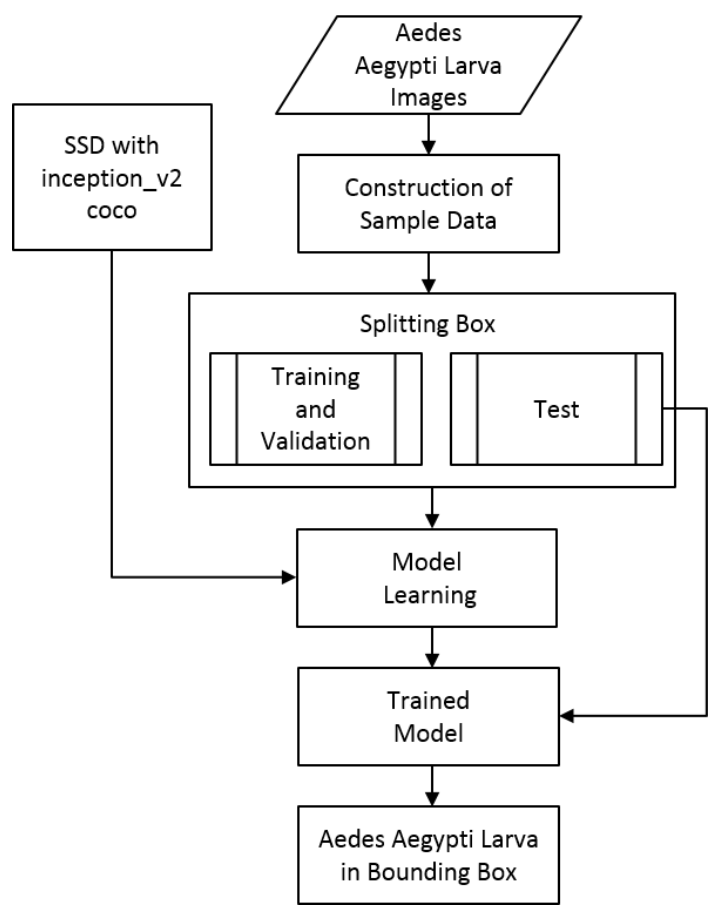

Figure 2. Flowchart of the experiment
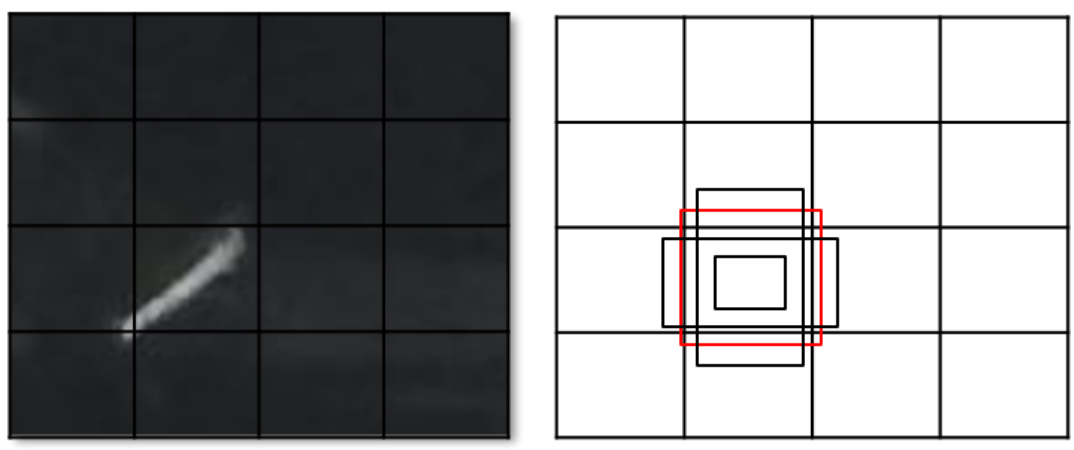

Figure 3. Default box working on detecting Aedes Aegypti larvae

In SSD framework, only input image and ground truth boxes were needed for detecting Aedes Aegypti during training. The box was evaluated in different aspect ratios at each location. In this case, feature maps of $4 \times 4$ scales is predicted the shape offsets and the confidence of Aedes Aegypti where the default box was treated as the positives and the rest as negatives. The cross-entropy loss is weighted sum between the confidence loss and localization loss. The overall training objective loss is defined as below [11]:

$$
L(x, c, l, g)=\frac{1}{N}\left(L_{\text {conf }}(x, c)+\alpha L_{l o c}(x, l, g)\right)
$$

where $\mathrm{N}$ is number of default boxes, $\mathrm{L}$ is localization loss, $\mathrm{c}$ is offset for center, 1 is predicted box and $\mathrm{g}$ is the ground truth box parameters.

\section{RESULTS AND ANALYSIS}

This section discusses the result on the detection retrained model. Figure 4 shows the training loss of the model. The result showed the unfiltered trained model. In the training, about 110k steps before were 
covered before the training stopped. Initially, the training phase declined drastically from beginning until $20 \mathrm{k}$ step before it declined evenly from 20k step until 80k step. Then the training loss was saturated at 1.5 from 80k step until the end of training which was 110k step. The final precision mAP@0.5IOU of the training was 0.8562. After the training, test image was implemented to evaluate the model. About 5 images containing 48 Aedes Aegypti larvae were used for the test trained model. The evaluation performance of Aedes Aegypti larvae included probability of detection $\mathrm{Pd}$ and probability of false alarm $\mathrm{Fa}$. The results are shown in Table 1.

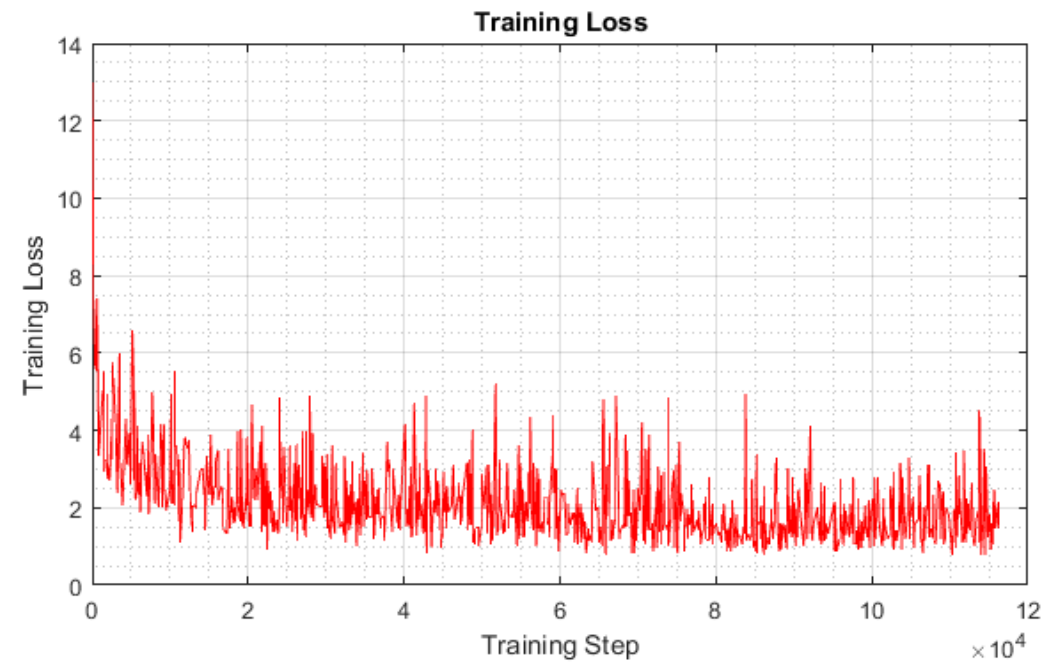

Figure 4. Training loss of the model

Table 1. Result of Aedes aegypti larvae test images

\begin{tabular}{cccccc}
\hline Model & $\mathrm{N}_{\text {total_larva }}$ & $\mathrm{N}_{\text {detect_larva }}$ & $\mathrm{N}_{\text {false larva }}$ & $\mathrm{P}_{\mathrm{d}}$ & $\mathrm{F}_{\mathrm{a}}$ \\
\hline $\mathrm{SSD}$ & 46 & 37 & 0 & 0.8043 & 0.0000 \\
\hline
\end{tabular}

The results showed that SSD performed about $80 \%$ accuracy in detecting Aedes Aegypti larvae. The loss of accuracies may bw attributed to the data fed during training because the Aedes Aegypti larvae was too small and may made the images too sparse for training. However, even the data used for Aedes Aegypti larvae detection were quite small, the overall test was still satisfactory and there was no false alarm in the test. Figure 5 shows the Aedes Aegypti larvae detection in water storage tank.
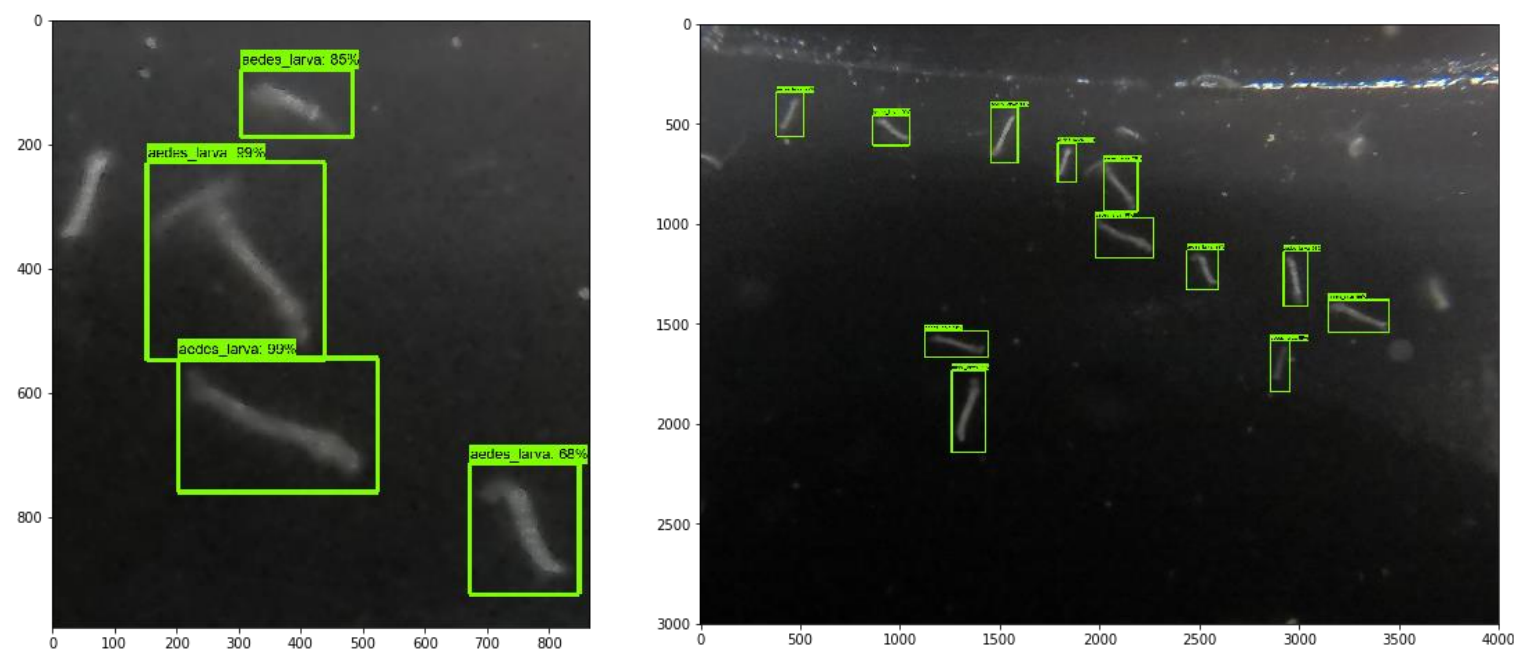

Figure 5. Aedes aegypti larvae detection in water storage tank 


\section{CONCLUSION}

To conclude, trained inference graph has successfully produced and trained to detect and localize Aedes Aegypti larvae in images. SSD is used with transfer learning to perform the Aedes Aegypti larvae detection. The results reveal that the model has achieved satisfactory probability of detection and performed better in probability of false alarm. Further study should focus on detecting small objecst in image to improve detection of Aedes Aegypti larvae.

\section{ACKNOWLEDGEMENTS}

This study is supported by a grant of Knowledge Transfer Program from Ministry of Higher Education (MoE), VOT number Gluar/KTP/2/2015/FKE/I/G00039. Sincere compliments to Faculty of Electrical Engineering (FKE), Universiti Teknikal Malaysia Melaka (UTeM) for its support. Deepest gratitude to Unit of Medical Entomology, Institute Medical for Research (IMR) in providing the training, research and related respective programs.

\section{REFERENCES}

[1] A. Krizhevsky, I. Sutskever, and H. Geoffrey E., "ImageNet Classification with Deep Convolutional Neural Networks," Adv. Neural Inf. Process. Syst. 25, pp. 1-9, 2012.

[2] B. Kay, "Dengue vector surveillance and control.," Curr. Opin. Infect. Dis., vol. 12, no. 5, pp. 48-59, 1999.

[3] R S. N. R. Saleeza, Y. Norma-Rashid, and M. S. Azirun, "Mosquitoes Larval Breeding Habitat in Urban and Suburban Areas , Peninsular Malaysia,” World Acad. Sci. Eng. Technol., vol. 58, no. 10, pp. 569-573, 2011.

[4] P. Sermanet, D. Eigen, X. Zhang, M. Mathieu, R. Fergus, and Y. LeCun, "OverFeat: Integrated Recognition, Localization and Detection using Convolutional Networks," 2013.

[5] R. Girshick, J. Donahue, T. Darrell and J. Malik, "Rich Feature Hierarchies for Accurate Object Detection and Semantic Segmentation," 2014 IEEE Conference on Computer Vision and Pattern Recognition, Columbus, OH, 2014, pp. 580-587.

[6] R. Girshick, "Fast R-CNN," 2015 IEEE International Conference on Computer Vision (ICCV), Santiago, 2015, pp. 1440-1448.

[7] S. Ren, K. He, R. Girshick and J. Sun, "Faster R-CNN: Towards Real-Time Object Detection with Region Proposal Networks," in IEEE Transactions on Pattern Analysis and Machine Intelligence, vol. 39, no. 6, pp. 1137-1149, 1 June 2017.

[8] J. Redmon, S. Divvala, R. Girshick and A. Farhadi, "You Only Look Once: Unified, Real-Time Object Detection," 2016 IEEE Conference on Computer Vision and Pattern Recognition (CVPR), Las Vegas, NV, 2016, pp. 779-788.

[9] J. Redmon and A. Farhadi, "YOLO9000: Better, Faster, Stronger," arXiv, 2016.

[10] J. Dai, Y. Li, K. He, and J. Sun, "R-FCN: Object Detection via Region-based Fully Convolutional Networks," in Conference: Advances in neural information processing systems, 2016, pp. 379-387.

[11] W. Liu, D. Anguelov, D. Erhan, C. Szegedy, S. Reed, C. Fu, and A. C. Berg, "SSD: Single Shot MultiBox Detector," in European conference on computer vision, 2016, pp. 21-37.

[12] A. Moore, "Artificial neural network trained to identify mosquitos in flight," J. Insect Behav., vol. 4, no. 3, pp. 391-395, 1991.

[13] F. Ibrahim, M. N. Taib, W. A. B. W. Abas, C. C. Guan, and S. Sulaiman, "A novel dengue fever (DF) and dengue haemorrhagic fever (DHF) analysis using artificial neural network (ANN)," Comput. Methods Programs Biomed., vol. 79, no. 3, pp. 273-281, 2005.

[14] H. M. Aburas, B. G. Cetiner, and M. Sari, "Dengue confirmed-cases prediction: A neural network model," Expert Syst. Appl., vol. 37, no. 6, pp. 4256-4260, 2010.

[15] B. G. Cetiner, M. Sari, and H. M. Aburas, "Recognition of Dengue Disease Patterns Using Artificial," 5th International Advanced Technologies Symposium (IATS'09), Karabuk, Turkey. May 13-15 2009, pp. 13-16.

[16] M. A. Mohd Fuad, M. R. Ab Ghani, R. Ghazali, M. F. Sulaima, M. H. Jali, T. Sutikno, T. A. Izzuddin, and Z. Jano, "A Review on Methods of Identifying and Counting Aedes Aegypti Larvae using Image Segmentation Technique," Telkomnika (Telecommunication Comput. Electron. Control), vol. 15, no. 3, pp. 1199-1206, 2017.

[17] Sumardi, M. Taufiqurrahman, and M. A. Riyadi, "Street mark detection using raspberry pi for self-driving system," TELKOMNIKA (Telecommunication Comput. Electron. Control), vol. 16, no. 2, pp. 629-634, 2018.

[18] D. Kurniawan, P. Adhi, A. Suryadi, I. Syamsu, and T. Praludi, "Object Detector on Coastal Surveillance Radar Using Two-Dimensional Order-Statistic Constant-False Alarm Rate Algoritm," TELKOMNIKA (Telecommunication Comput. Electron. Control), vol. 13, no. 2, p. 624-631, 2015.

[19] M. A. Mohd Fuad, M. R. Ab Ghani, R. Ghazali, T. A. Izzuddin, M. F. Sulaima, Z. Jano and T. Sutikno, "Training of Convolutional Neural Network using Transfer Learning for Aedes Aegypti Larvae," TELKOMNIKA (Telecommunication Computing Electronics and Control), vol. 16, no. 4, p. 1894-1900, 2017. 